\title{
Aquanet Bowel Cleansing Device Versus Oral Sodium Picosulfate for Pre-Endoscopy Bowel Preparation: Propensity Score Analysis for Interventional Effectiveness Evaluation
}

\author{
Roberto L. Kaiser Juniora, c, Luiz G. de Quadros ${ }^{\text {a, b }}$, Mikaell A.G. Faria ${ }^{a}$, \\ Fernanda S.L. Kaisera , Juan C.O. Campo ${ }^{a}$, \\ Idiberto J. Zotarelli Filho ${ }^{\text {a }}$
}

\begin{abstract}
Background: Colonoscopy procedures are commonly performed and have high success rates. However, poor or inadequate bowel preparation is one of the most common reasons for a repeated or failed colonoscopy. We therefore performed an observational study followed by propensity score modeling to evaluate and compare the quality of bowel preparation with the use of Aquanet bowel cleansing devices (BCDs) versus the use of oral sodium picosulfate solution.

Methods: We performed a prospective cross-sectional study to compare the quality of pre-endoscopic bowel preparation using a BCD with oral solution. Our major outcome of interest was the quality of bowel preparation as measured through the Boston bowel preparation (BBP) scale. Our main predictor was the method of bowel preparation. The bowel was prepared using either sodium picosulfate or the BCD.
\end{abstract}

Results: A total of 314 participants were part of this study. The average age of the participants was 54 years and most of the participants were females $(81 \%)$. Sodium picosulfate was associated with better scores at each segment. After propensity scoring with a 1:1 match and further adjusting for the unbalanced variable (age), we found that despite the apparent superior cleansing performance of sodium picosulfate over the $\mathrm{BCD}$, the difference was not statistically significant.

Conclusion: This study reinforces previous reports regarding the quality, safety and comfort of BCDs, indicating that this technique should be considered for colonoscopy preparation. In the future, randomized controlled trials should be performed to validate these pre-

Manuscript submitted December 7, 2017, accepted December 27, 2017

aKaiser Clinic and Day Hospital, Sao Jose do Rio Preto, SP, Brazil

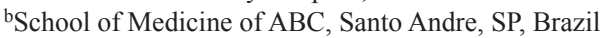

${ }^{\mathrm{c} C o r r e s p o n d i n g ~ A u t h o r: ~ R o b e r t o ~ L u i z ~ K a i s e r ~ J u n i o r, ~ K a i s e r ~ C l i n i c ~ a n d ~ D a y ~}$ Hospital, Street XV de Novembro, 3975, Redentor Sao Jose do Rio Preto, SP, Brazil. Email: kaiserjr@kaiserclinica.com.br

doi: https://doi.org/10.14740/gr942w liminary findings.

Keywords: Aquanet; Sodium picosulfate; Bowel cleansing; Propensity score; Clinical study

\section{Introduction}

Colonoscopy is vital for the diagnosis of colorectal diseases, being the current gold standard method for investigating the colonic, rectal, and terminal ileal mucosa [1]. This includes the entire mucosa from the anal margin to the ileocecal valve, and is particularly important in the context of screening/surveillance for colorectal cancer (CRC). This is relevant as colorectal cancer is a major cause of mortality worldwide, with an incidence of approximately 900,000 cases per year [2,3]. Mortality and morbidity from colorectal cancer can be decreased if screening is performed on all individuals above the age of 50 . Colonoscopy is not just a screening tool, but also plays a role in the diagnosis of proximally located colonic cancers which will not be detected with simple sigmoidoscopy [4].

Apart from the use of enemas and laxatives for bowel cleansing, devices specifically manufactured for cleansing have also been developed. One such device, ColonoScoPrep ${ }^{\mathrm{TM}}$ (Aquanet), was designed to use triple filtered water, carbon microsediments and ultraviolet light, while working within a fixed pressure and gravity system, thereby increasing intestinal washing efficiency [5]. It has been demonstrated that bowel cleansing devices (BCDs) such as Jetprep (Jetprep LLC, Herzliya, Israel), Medjet, and ColonoScoPrep ${ }^{\mathrm{TM}}$ improve intestinal cleansing, are safe, effective, and well tolerated by patients [5-7].

Colonoscopy procedures are commonly performed with high success rates. However, poor or inadequate bowel preparation is one of the most common reasons for a repeated or failed colonoscopy [8]. Inadequate bowel cleansing also has a negative impact on cecal intubation success rates, decreasing polyp detection and retrieval rates, increasing the time of 
the overall procedure, and ultimately affecting not only its effectiveness but also its safety [7, 8]. Factors predicting inadequate preparation include stroke, dementia, opioid use, calcium-channel blockers and anti-depressants [9]. In contrast, an adequately prepared colon enables a full view of the mucosa, hence reducing the rates of repeated examinations and also decreasing the rates of endoscopists missing lesions.

The success of a colonoscopy procedure is dependent on the effectiveness of its preparation. Preparation methods shown to be effective include the use of either BCDs or oral laxatives. The use of cleansing methods such as enemas and laxatives is important in increasing the sensitivity of the colonoscopy procedures. Most enemas have their causal mechanisms related to rectal distension and mucosa irritation [10, 11]. Commonly used bowel preparation enema and laxative methods include polyethylene glycol (PEG) and sodium picosulfate solution (Picoprep), respectively $[12,13]$.

The use of polyethylene glycol (PEG) has been associated with numerous adverse effects including nausea, vomiting, bloating and abdominal cramps [10]. On the other hand, sodium picosulfate solution, a combination of magnesium oxide and citric acid, manufactured by Ferring Pharmaceuticals, Parsippany, NJ, has been demonstrated to have fewer adverse effects. It is an effective, low volume bowel cleansing product with excellent effectiveness, adequate tolerance, and less electrolyte imbalance compared to PEG $[10,14]$.

Despite the acceptable effectiveness and safety of both bowel cleansing methods, very few studies have been performed to test which method is more effective. Specifically, to the best of our knowledge, no study has been conducted to evaluate and compare the quality of bowel preparations using different oral-cleansing solutions versus BCDs. We therefore performed an observational study followed by propensity score modeling to evaluate and compare the quality of bowel preparation with the use of Aquanet BCDs versus the use of oral sodium picosulfate solution.

\section{Methods}

\section{Study design}

We performed a prospective cross-sectional study to compare the quality of pre-endoscopic bowel preparation using a BCD with oral sodium picosulfate solution. A total of 314 participants were part of our study, and the study was described in accordance with the STROBE (Strengthening the Reporting of Observational studies in Epidemiology) guidelines [15].

\section{Ethics}

This study was evaluated and approved by the Research Ethics Committee of the Portuguese Charitable Hospital (protocol number 655036, May 19, 2014) in Sao Jose do Rio Preto, SP, Brazil, and informed consent was offered to all potential participants and subsequently signed prior to any study protocol being implemented.

\section{Setting}

Data collection was performed from June 2014 to February 2015 at the Portuguese Charitable Hospital, located at Sao Jose do Rio Preto, SP, Brazil. Patients came from Sao Paulo state. The Kaiser clinic, a private institution, paid for the Aquanet devices. The Aquanet devices were designed and tested by Prime Pacific Health Innovations Corporation (http://www.primepacifichealth.com/aquanet-family-of-colon-hydrotherapy-devices/, last accessed December 2016), and approved by the various regulatory authorities for market approval and licensing including FDA, Health Canada, and EU (http://www.accessdata.fda.gov/scripts/cdrh/cfdocs/ cfPMN/pmn.cfm?ID=K000031, last accessed December 2016). The procedure was inexpensive and the process lasted 40 - $60 \mathrm{~min}$. It makes use of filtered, ultraviolet treated water and derives the water pressure from gravity or from a pump with an upper safe limit of 2 per square inch (psi) of pressure. The device cleanses the large bowel through a series of fill and empty cycles by hydrating the colon, stimulating peristalsis and evacuating bowel contents. The process is intended to hydrate the colon without a significant loss of electrolytes. Although no significant adverse events have been reported as of December of 2016, the device may cause mild abdominal discomfort during the water infusion phase in some patients.

\section{Participants}

Our participants were selected among all patients requiring a colonoscopy. We included those between the ages of 14 and 90 years, and who also presented more than three bowel movements per week for the past 1 month. We excluded those who had the following conditions: pregnancy (confirmed by a pregnancy test), acute abdomen syndrome, prior colorectal surgery, hemorrhoids or endoscopic procedures, known bowel diseases (colon cancer history, toxic megacolon, toxic colitis, idiopathic pseudo obstruction, hypo-motility syndrome), other gastrointestinal disorders (active ulcer, output obstruction, retention, gastroparesis, ileus), previous upper gastrointestinal surgery (gastrectomy, gastric band, gastric bypass), uncontrolled angina and/or myocardial infarction (MI) within the last 3 months, congestive heart failure (CHF) or uncontrolled hypertension, renal impairment (serum, creatinine and potassium must be within normal limits) or known hypersensitivity to active ingredients.

\section{Outcome variables}

Our major outcome of interest was the quality of bowel preparation as measured through the Boston bowel preparation (BBP) scale. Bowel preparation was evaluated in three segments: the right segment (cecum and ascending colons), transverse segment (transverse colon including liver and splenic angles), and the left segment (descending and sigmoid colons and rectum). Each segment was evaluated on a scale from 0 to 3, 
Table 1. Baseline Characteristics of Participants Stratified by Bowel Preparation

\begin{tabular}{lllll}
\hline Variable & Total (314) & Aquanet (157) & Sodium picosulfate (157) & P \\
\hline Age & $54.22 \pm 17.61$ & $58.2 \pm 16.87$ & $50.24 \pm 17.48$ & $<0.001$ \\
Gender (female) & $254(80.9 \%)$ & $143(91.1 \%)$ & $111(70.7 \%)$ & 0.001 \\
Intestinal symptoms & & & $31(19.7 \%)$ & 0.017 \\
$\quad$ Constipated & $46(14.6 \%)$ & $15(9.6 \%)$ & $126(80.3 \%)$ & \\
\hline \multicolumn{1}{c}{ Normal } & $268(85.4 \%)$ & $142(90.4 \%)$ & & \\
\hline
\end{tabular}

and the final score for an individual was the sum of scores for all three segments. Using this score, 0 was the minimum score corresponding to an unprepared colon and 9 was the maximum score corresponding to an excellent preparation without any residual traces [16]. Inadequate preparation corresponded to a BBP score of less than $5[17,18]$.

\section{Predicting variables}

Our main predictor was the method of bowel preparation. The bowel was prepared using either sodium picosulfate or the $\mathrm{BCD}$. On the day prior to the colonoscopy, all participants on sodium picosulfate took four tablets of Dulcolax with tea or water in the morning, liquid diet (juice, tea or water) at lunch, two capsules of $25 \mathrm{mg}$ Dramamine Capsgel in the afternoon, sodium picosulfate dissolved in $150 \mathrm{~mL}$ of cold water $30 \mathrm{~min}$ after, followed by drinking at least five $250 \mathrm{~mL}$ cups of water or other light liquids till midnight, with absolute fasting until the colonoscopy was performed. For bowel preparation with the $\mathrm{BCD}$, intestinal lavage was performed with the device, and made use of water, pressure, and gravity to enhance bowel cleansing. The water used in this procedure was previously triple-filtered by its passage on carbon, micro-pellets and ultraviolet light. The preparation was carried out by a trained nurse. All endoscopies were performed by an endoscopist who was blinded to the method of preparation.

\section{Potential confounding variables}

Our potential confounders were selected based on evidence from previous literature combined with our clinical judgment. Specifically, we selected age and gender as potential confounders [19].

\section{Statistical methods}

Our exploratory analysis was started by evaluating distributions, frequencies and percentages for each of the numeric and categorical variables. Categorical variables were evaluated for near-zero variation [20]. Extensive graphical displays were used for both univariate analysis and bivariate associations, accompanied by broader tests such as maximal information coefficient [21] and non-negative matrix factorization [22] algorithms for numeric variables. Missing data were ex- plored using a combination of graphical displays involving univariate, bivariate and multivariate methods. Imputation was performed using a k-nearest neighbors algorithm $(n=5)$ [23].

The association between bowel cleansing procedures and quality of bowel cleansing was evaluated using propensity matching scores. The analysis was started by building propensity scores using the bowel cleansing procedure as a dependent variable and our previously listed potential confounders as predictors. Propensity scores $($ alpha $=20)$ were then matched between the two arms of our interventions, their balance evaluated through a combination of plots as well as statistical tests, specifically $t$-tests and Chi-square tests. Once matched controls were found, comparisons were conducted between Aquanet vs. sodium picosulfate procedures in predicting quality of bowel cleansing. Variables which did not balance after matching were further adjusted for in a generalized linear model [24].

All analyses were performed using the R language [22] and the following packages: ggplot2, rmarkdown, and nonrandom.

\section{Results}

\section{Participants}

A total of 314 participants were part of this analysis. Average age was 54 years old, with most participants being females $(81 \%)$. Some of the participants had constipation (14.6\%) (Table 1).

\section{Outcomes}

When evaluating the quality of bowel cleansing by intervention and then at each segment, we observed that bowel cleansing was associated with better scores in the distal colon compared to the proximal colon for both methods. The distal colon was associated with the worse cleansing scores (Table 2). In addition, sodium picosulfate was more effective with better total scores $(7.28 \pm 1.95,88.5 \%)$ when compared to a BCD $(6.02$ $\pm 2.51,68.8 \%)$.

\section{Propensity score matching}

After a 1:1 matching, groups were tested for balance for each 
Table 2. Boston Scores for Bowel Segments Stratified by Bowel Preparation

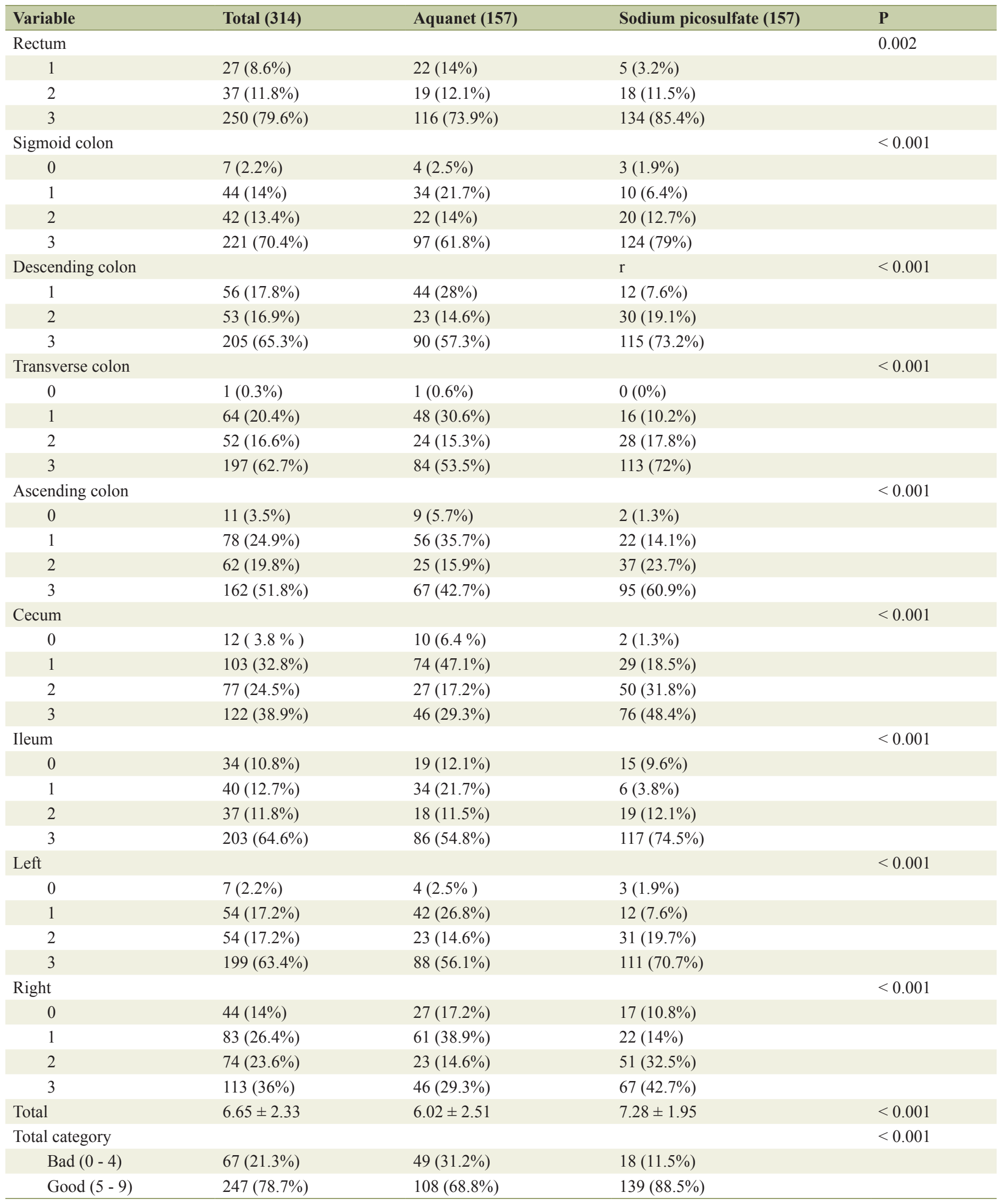




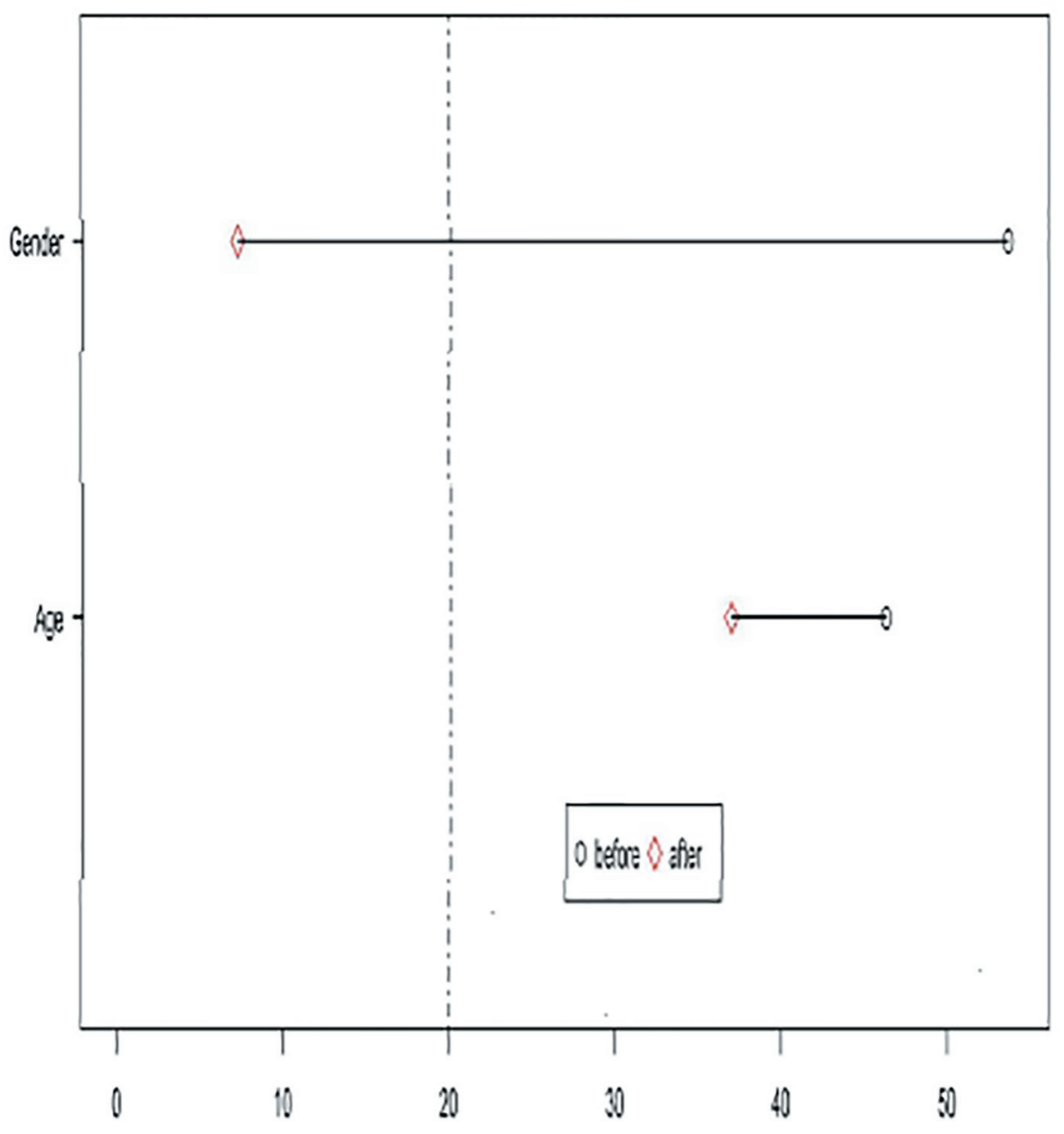

Figure 1. Testing for balance after matching.

covariate using standard differences with an alpha of 20. While gender was balanced after matching, age remained unbalanced (Fig. 1).

\section{Outcomes evaluation after propensity score matching}

When evaluating outcomes through propensity scores with a 1:1 match for the treatment (Aquanet) group, and then further adjusting for the unbalanced variable (age), we found that despite the apparent superior cleansing performance of sodium picosulfate over the BCD, the difference was not statistically

Table 3. Predicted Boston Scores: Crude Scores, Scores After Propensity Matching and After Adjusting for Age

\begin{tabular}{lll}
\hline Model & Sodium picosulfate & $\begin{array}{l}\text { Aquanet } \\
\text { (bowel cleansing device) }\end{array}$ \\
\hline Crude & $7.28(6.93,7.63)$ & $6.02(5.52,6.52)$ \\
Unadjusted & $7.16(6.76,7.55)$ & $5.92(5.39,6.45)$ \\
Adjusted & $8.6(7.66,9.53)$ & $7.54(7,8.08)$ \\
\hline
\end{tabular}

significant (Table 3).

\section{Discussion}

To our knowledge, this is the first study to compare the quality of bowel preparation for colonoscopy using a BCD (Colon$\mathrm{oScoPrep}^{\mathrm{TM}}$ or Aquanet) versus sodium picosulfate oral solution. Our analyses demonstrated that the device was as effective as the oral preparation when evaluated through the BBP scale, with maximum adjusted scores being 7.5 and 8.6, respectively (all scores with $\mathrm{P}>0.05$ ).

Our results are aligned to previous reports on the effectiveness of both techniques on bowel cleansing $[6,10,25]$. As the level of effectiveness was similar between both methods, tolerability and safety must be decisive factors when making a choice [26]. Poorly tolerated methods tend to not be performed according to their instructions especially when used at home by patients, resulting in inadequate bowel preparation [27].

Sodium picosulfate is a stimulant laxative associated with abdominal discomfort, risk of electrolyte alterations and dehydration [28]. In contrast, ColonoScoPrep ${ }^{\mathrm{TM}}$ is a water-based 
filtration system, providing not only an efficient and safe intestinal washing along with high satisfaction and comfort levels by patients, but has also been associated with minimal changes in electrolyte levels $[5,29]$. Hence, ColonoScoPrep ${ }^{\mathrm{TM}}$ might be suitable for patients vulnerable to hydro-electrolytic imbalance. BCDs however, are not recommended for patients with inflammatory bowel disease, perianal disease or obstructive symptoms [5].

BCDs present a number of other advantages. First, the procedure can be performed right before the colonoscopy, which is convenient for patients, only requiring the use of laxatives on the preceding night [15]. Sodium picosulfate in contrast, demands three intakes of a laxative solution as well as a restrictive diet during the day prior to the colonoscopy [30]. Given the ease in performing the exam with BCDs, the adherence to the procedure may be improved. As a consequence, when considering both procedures from a healthcare policy perspective, ColonoScoPrep ${ }^{\mathrm{TM}}$ might lead to a potential decrease in morbimortality, with costs justifying the indication of this device as an attractive choice for colonoscopy preparation [5].

Despite filling an important gap in the literature, our study does have limitations. First, the propensity score does not balance unmeasured confounding variables as would be the case with a randomized controlled trial. Second, although we attempted to be as inclusive as possible in our criteria and recruitment strategies, interventional studies tend to over-select patients who, compared to the general population, do not have as many comorbidities, are more educated, are not as representative of minority groups, and have a greater income. Despite these limitations, we believe that the internal validity provided through our propensity score-matching establishes an important new ground of evidence in the field.

In conclusion, this study reinforces previous reports regarding the quality of BCDs, indicating that this technique should be considered for colonoscopy preparation. In the future, randomized controlled trials should be performed to validate these preliminary findings.

\section{Acknowledgments}

We received statistical and English reviewing assistance from SporeData Inc.

\section{Author Contributions}

Dr Kaiser Junior had full access to all of the data in the study and takes responsibility for the integrity of the data and the accuracy of the data analysis. Conception and design: Roberto Luiz Kaiser Junior, Luiz Gustavo de Quadros, and Mikaell Alexandre Gouvea Faria. Analysis and interpretation: Luiz Gustavo de Quadros and Fernanda Soubhia Liedtke Kaiser. Drafting of the manuscript: Roberto Luiz Kaiser Junior, Luiz Gustavo de Quadros, Fernanda Soubhia Liedtke Kaiser, Juan Carlos Ochoa Campo, and Idiberto Jose Zotarelli Filho. Reviewing: Roberto Luiz Kaiser Junior, Luiz Gustavo de Quadros, and Mikaell anAlexandre Gouvea Faria. Final approval of the manuscript: Roberto Luiz Kaiser Junior.

\section{Funding Support}

None.

\section{References}

1. Winawer S, Fletcher R, Rex D, Bond J, Burt R, Ferrucci J, Ganiats T, et al. Colorectal cancer screening and surveillance: clinical guidelines and rationale-Update based on new evidence. Gastroenterology. 2003;124(2):544-560.

2. Fayad NF, Kahi CJ. Colonoscopy quality assessment. Gastrointest Endosc Clin N Am. 2015;25(2):373-386.

3. Boyle P, Leon ME. Epidemiology of colorectal cancer. $\mathrm{Br}$ Med Bull. 2002;64:1-25.

4. Lieberman DA, Weiss DG, Bond JH, Ahnen DJ, Garewal $\mathrm{H}$, Chejfec G. Use of colonoscopy to screen asymptomatic adults for colorectal cancer. Veterans Affairs Cooperative Study Group 380. N Engl J Med. 2000;343(3):162168.

5. Ziv Y, Scapa E. A new colonic lavage system to prepare the colon for colonoscopy: a retrospective study. Tech Coloproctol. 2013;17(1):39-44.

6. Eliakim R, Yassin K, Lachter J, Chowers Y. A novel device to improve colon cleanliness during colonoscopy. Endoscopy. 2012;44(7):655-659.

7. Rigaux J, Juriens I, Deviere J. A novel system for the improvement of colonic cleansing during colonoscopy. Endoscopy. 2012;44(7):703-706.

8. Butt SK, Defoe H, Besherdas K. PWE-200 The impact of bowel preparation on other colonoscopy quality indicators. Gut. 2012;61:A378.

9. Yee R, Manoharan S, Hall C, Hayashi A. Optimizing bowel preparation for colonoscopy: what are the predictors of an inadequate preparation? Am J Surg. 2015;209(5):787792; discussion 792.

10. Kojecky V, Dolina J, Kianicka B, Misurec M, Varga M, Latta J, Vaculin V. A single or split dose picosulphate/magnesium citrate before colonoscopy: comparison regarding tolerance and efficacy with polyethylene glycol. A randomized trial. J Gastrointestin Liver Dis. 2014;23(2):141146.

11. Portalatin M, Winstead N. Medical management of constipation. Clin Colon Rectal Surg. 2012;25(1):12-19.

12. Flemming JA, Vanner SJ, Hookey LC. Split-dose picosulfate, magnesium oxide, and citric acid solution markedly enhances colon cleansing before colonoscopy: a randomized, controlled trial. Gastrointest Endosc. 2012;75(3):537-544.

13. Seo EH, Kim TO, Park MJ, Joo HR, Heo NY, Park J, Park $\mathrm{SH}$, et al. Optimal preparation-to-colonoscopy interval in split-dose PEG bowel preparation determines satisfactory bowel preparation quality: an observational prospective study. Gastrointest Endosc. 2012;75(3):583-590.

14. Gweon TG, Kim SW, Noh YS, Hwang S, Kim NY, Lee Y, Lee SW, et al. Prospective, randomized comparison of 
same-day dose of 2 different bowel cleanser for afternoon colonoscopy: picosulfate, magnesium oxide, and citric acid versus polyethylene glycol. Medicine (Baltimore). 2015;94(13):e628.

15. von Elm E, Altman DG, Egger M, Pocock SJ, Gotzsche PC, Vandenbroucke JP, Initiative S. The Strengthening the Reporting of Observational Studies in Epidemiology (STROBE) statement: guidelines for reporting observational studies. PLoS Med. 2007;4(10):e296.

16. Lai EJ, Calderwood AH, Doros G, Fix OK, Jacobson BC. The Boston bowel preparation scale: a valid and reliable instrument for colonoscopy-oriented research. Gastrointest Endosc. 2009;69(3 Pt 2):620-625.

17. Kim ES, Lee WJ, Jeen YT, Choi HS, Keum B, Seo YS, Chun HJ, et al. A randomized, endoscopist-blinded, prospective trial to compare the preference and efficacy of four bowel-cleansing regimens for colonoscopy. Scand J Gastroenterol. 2014;49(7):871-877.

18. Kim EJ, Park YI, Kim YS, Park WW, Kwon SO, Park $\mathrm{KS}$, Kwak CH, et al. A Korean experience of the use of Boston bowel preparation scale: a valid and reliable instrument for colonoscopy-oriented research. Saudi J Gastroenterol. 2014;20(4):219-224.

19. Lee PH. Should we adjust for a confounder if empirical and theoretical criteria yield contradictory results? A simulation study. Sci Rep. 2014;4:6085.

20. Morris DH, Gostic KM, Pompei S, Bedford T, Luksza M, Neher RA, Grenfell BT, et al. Predictive modeling of influenza shows the promise of applied evolutionary biology. Trends Microbiol. 2018;26(2):102-118.

21. Reshef DN, Reshef YA, Finucane HK, Grossman SR, McVean G, Turnbaugh PJ, Lander ES, et al. Detecting novel associations in large data sets. Science. 2011;334(6062):1518-1524.

22. Paatero P, Tapper U. Positive matrix factorization: A nonnegative factor model with optimal utilization of error estimates of data values. Environmetrics. Wiley Online Library. 1994;5:111-126.

23. Prantner B. Visualization of imputed values using the $r-$ package vim. 2011.

24. R Core Team. R: A language and environment for statistical computing [Internet]. Vienna, Austria: R Foundation for Statistical Computing; 2015. Available from: http:// www.R-project.org/.

25. Kiesslich R, Schuster N, Hoffman A, Goetz M, Galle PR, Santo E, Halpern Z. MedJet - a new CO2-based disposable cleaning device allows safe and effective bowel cleansing during colonoscopy: a pilot study. Endoscopy. 2012;44(8):767-771.

26. Barkun A, Chiba N, Enns R, Marcon M, Natsheh S, Pham C, Sadowski D, et al. Commonly used preparations for colonoscopy: efficacy, tolerability, and safety-a Canadian Association of Gastroenterology position paper. Can J Gastroenterol. 2006;20(11):699-710.

27. Johnson DA, Barkun AN, Cohen LB, Dominitz JA, Kaltenbach T, Martel M, Robertson DJ, et al. Optimizing adequacy of bowel cleansing for colonoscopy: recommendations from the US multi-society task force on colorectal cancer. Gastroenterology. 2014;147(4):903-924.

28. Navarro A, Hession PT. Efficacy and tolerability of sodium picosulphate/magnesium citrate as a bowel-cleansing agent - Results from a literature review. Eur Gastroenterol Hepatol Review. 2009;5:11-14.

29. P CJM. Effects of colon irrigation on serum electrolytes ozone therapy articles at VitalityLink.com [Internet]. VitalityLink. [cited 2016 Mar 23]. Available from: http:// www.vitalitylink.com/article-colonic-hydrotherapy1672-effects-colon-irrigation-serum-electrolytes-http.

30. Schmidt LM, Williams P, King D, Perera D. Picoprep-3 is a superior colonoscopy preparation to Fleet: a randomized, controlled trial comparing the two bowel preparations. Dis Colon Rectum. 2004;47(2):238-242. 